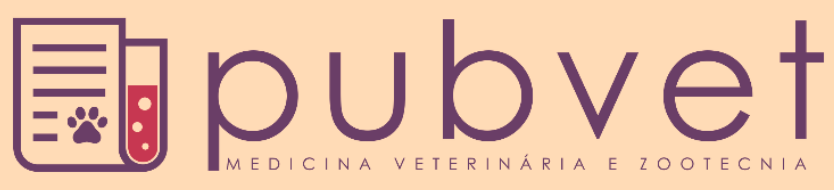

https://doi.org/10.31533/pubvet.v14n10a666.1-5

\title{
Pseudocisto perirenal extracapsular em um gato: Relato de caso
}

\author{
Joyce Marina Francisco de Moraes ${ }^{1,2}$, Cairbar Euripedes de Moraes ${ }^{1}$, Carlos Eduardo Moura \\ Delatore $^{1}$, Karla Menezes Cardoso ${ }^{3,5,69}$, Tarcísio Guerra Guimarães ${ }^{3,4,5,6 *}$ () \\ ${ }^{1}$ Clínica Veterinária Toca dos Bichos, Pitangueiras-SP, Brasil. \\ ${ }^{2}$ Discente do Programa de Pós Graduação Veterinária, Equalis, São Paulo-SP, Brasil. \\ ${ }^{3}$ Instituto de Investigação e Formação Avançada (IIFA), Universidade de Évora, Portugal. \\ ${ }^{4}$ Instituto de Ciências Agrárias e Ambientais Mediterrânicas (ICAAM), Universidade de Évora, Portugal. \\ ${ }^{5}$ Centro Acadêmico Clínico de Coimbra (CACC), Coimbra, Portugal. \\ ${ }^{6}$ Instituto de Investigação Clínica e Biomédica (iCBR), área de Meio Ambiente, Genética e Oncobiologia (CIMAGO), Faculdade de Medicina, \\ Universidade de Coimbra, Portugal. \\ *Autor para correspondência, E-mail: tarcisiounifran@yahoo.com.br; tarcisioguerra77@gmail.com
}

Resumo. Pseudocisto perirenal é uma desordem pouco relatada em animais domésticos. Caracteriza-se pelo acúmulo de fluido em um saco fibroso ao redor de um ou dos dois rins. Quando o fluido acumula-se entre a cápsula renal e a parede peritoneal é classificado como extracapsular ou retroperitoneal. Objetivou-se relatar o caso de um gato sem raça definida de dois anos de idade, atendido com queixa de distensão abdominal e anorexia. O exame ecográfico revelou imagens compatíveis com pseudocisto perirenal, demostrando grande volume de fluido envolvendo ambos os rins. $\mathrm{O}$ tratamento instituído foi através do procedimento de capsulotomia total com omentalização. O procedimento capsulotomia, demostrou ser um procedimento muito eficaz, sem a presença complicações ou recidivas, na resolução cirúrgica do pseudocisto perirenal extracapsular com acúmulo de transudato, sem causa aparente.

Palavras chave: bilateral, capsulotomia, felino, retroperitoneal

\section{Extracapsular perirenal pseudocyst in a cat: Case report}

Abstract. Perirenal pseudocyst is a disorder rarely reported in domestic animals. It is characterized by the accumulation of fluid in a fibrous sac around one or both kidneys. When fluid accumulates between the renal capsule and the peritoneal wall, it is classified as extracapsular or retroperitoneal. The objective was to report the case of a two-year-old mixed breed cat, attended with complaints of bloating and anorexia. The ultrasound examination revealed images compatible with perirenal peseudocyst, showing a large volume of fluid involving both kidneys. The treatment instituted was through the total capsulotomy procedure with omentalization. The capsulotomy procedure proved to be a very effective procedure, without the presence of complications or recurrences, in the surgical resolution of the extracapsular perirenal pseudocyst with accumulation of transudate, with no apparent cause.

Keywords: bilateral, capsulotomy, feline, retroperitoneal

\section{Pseudoquiste perirrenal extracapsular en un gato: Reporte de un caso}

Resumen. El pseudoquiste perirrenal es un trastorno raramente reportado en animales domésticos. Se caracteriza por la acumulación de líquido en un saco fibroso alrededor de uno o ambos riñones. Cuando se acumula líquido entre la cápsula renal y la pared peritoneal, se clasifica como extracapsular o retroperitoneal. El objetivo fue informar el caso de un gato mestizo de dos años, atendido con quejas de distensión abdominal y 
anorexia. El examen de ultrasonido reveló imágenes compatibles con el pseudoquiste perirrenal, mostrando un gran volumen de líquido que afecta a ambos riñones. El tratamiento instituido fue a través del procedimiento de capsulotomía total con omentalización. El procedimiento de capsulotomía demostró ser un procedimiento muy eficaz, sin la presencia de complicaciones o recurrencias, en la resolución quirúrgica del pseudoquiste perirrenal extracapsular con acumulación de transudado, sin causa aparente.

Palabras clave: bilateral, capsulotomia, felino, retroperitoneal

\section{Introdução}

O pseudocisto perirenal extracapsular ou retroperitoneal caracteriza-se pelo acúmulo de fluido em um saco fibroso ao redor do rim, localizado entre a cápsula renal e a parede peritoneal (Beck et al., 2000; Mutinelli et al., 2005). Quando esse acúmulo de fluido ocorre entre o parênquima e a cápsula renal, é considerado pseudocisto perirenal subcapsular (Placer \& McManis, 2019). O fluido pode acumular-se em um ou em ambos os rins (Beck et al., 2000), sendo a natureza do fluido do pseudocisto não é constante, podendo ser urina, linfa, sangue ou transudato (Essman et al., 2000).

O pseudocisto perirenal é uma doença incomumente relatada, podendo acometer os felinos (Beck et al., 2000), além de outras espécies (Miles \& Jergens, 1992; Puerto et al., 1998). A etiologia pode ser multifatorial (Ochoa et al., 1999), além de ocorrer sem causa aparente (Castro et al., 2013; Placer \& McManis, 2019). A apresentação clínica dessa desordem podem incluir distensão abdominal, vômito, perda de peso e anorexia (Schaefer et al., 2018).

A ecografia é uma ferramenta importante para a detecção de distúrbios renais e pode direcionar ao diagnóstico de forma precisa, revelando acúmulo de líquido em região perirenal (Debruyn et al., 2012). O tratamento empregado pode ser através da drenagem do conteúdo do pseudocisto, capsulotomia e omentalização (Hill \& Odesnik, 2000; McCord et al., 2008; Placer \& McManis, 2019). O presente trabalho tem como objetivo relatar a resolução e evolução clínica de gato com pseudocisto perirenal extracapsular, submetidos ao procedimento de capsulotomia total.

\section{Relato de caso}

Foi atendido, na Clínica Veterinária Toca dos Bichos, um gato, macho castrado, 2 anos, sem raça definida, pesando $4,2 \mathrm{~kg}$, com queixa de apatia e anorexia aproximadamente 3 dias. No exame clínico observou de forma evidente abdômen distendido (Figura 1A), com presença de massa firme, localizada na região cranial do abdômen (Figura 1B) e sem sensibilidade na palpação. Ao realizar o teste da onda liquida, observou transmissão positiva.

A ecográfia abdominal revelou a presença de grande conteúdo anecoico (fluido) envolvendo de forma bilateral os rins (Figura 2), sendo a imagem compatível com pseudocisto perirenal. A laparatomia exploratória foi indicada. O painel de avaliação hematológica e bioquímica, revelou o aumento da creatinina e ureia, demais exames apresentavam-se dentro dos valores de referência. $\mathrm{O}$ acesso venoso foi realizado e a fluidoterapia instituída. O protocolo anestésico consistiu, administração de tiletamina associada à zolazepam (Zoletil ${ }^{\circledR}$ ) na dose de $10 \mathrm{mg} / \mathrm{kg}$ e tramadol (Hipolabor ${ }^{\circledR}$ ) na dose de $4 \mathrm{mg} / \mathrm{kg}$ por via intramuscular (IM). A indução anestésica foi realizada com propofol (Propovan ${ }^{\circledR}$ ) por via intravenosa (IV) na dose de $5 \mathrm{mg} / \mathrm{kg}$, administrado em bolus (1/4 da dose calculada), seguido de intubação orotraqueal e manutenção anestésica com isofluorano (Isofurine ${ }^{\circledR}$ ), dose/efeito vaporizado em oxigênio $200 \mathrm{~mL} / \mathrm{kg} / \mathrm{min}$ em circuito semiaberto.

Após a celiotomia mediana, visualizou uma grande estrutura cística delimitada ao redor do rim direito (Figura 3A). A capsula cística apresentava aspecto fino, translucida e vascularizada. $\mathrm{O}$ rim esquerdo apresentava cisto de menor dimensão (Figura 3B), com as mesma características e aspecto. Demais órgãos não apresentaram alteração macroscópica dignas de nota. Foi realizada a centese asséptica dos cistos, com auxílio de seringas de $20 \mathrm{~mL}$ e agulha de 25 x 0,7mm. O conteúdo cístico, apresentava característica límpida de coloração amarelo claro que encaminhada para análise laboratorial. Foi realizada a incisão da capsula cística e visualização macroscópica dos rins que apresentavam envoltos pela capsula renal de aspecto fisiológico, caracterizando por pseudocisto perirenal extracapsular. Foi realizada a capsulotomia total bilateral, com a omentalização. A lavagem da cavidade peritoneal foi 
realizada com solução fisiológica estéril morna e em ato contínuo sutura muscular, subcutânea e cutânea foram confeccionadas.

Após a capsulotomia do pseudocisto, o animal foi mantido em ambiente hospitalar, recebeu fluido parenteral, amoxicilina + ácido clavulânico $\left(\right.$ Clavacillin $\left.^{\circledR}\right) 8,75 \mathrm{mg} /$ dia por via subcutânea (SC) a cada 24 horas, tramadol na dose de $2 \mathrm{mg} / \mathrm{kg} \mathrm{SC}$ a cada 12 horas e robenacoxibe (Onsior ${ }^{\circledR}$ ) na dose de $2 \mathrm{mg} / \mathrm{kg}$ $\mathrm{SC}$ a cada 24 horas. A função renal foi avaliada (ureia e creatinina) e encontravam-se nos parâmetros normais. Na ecografia de controle não foi observado a presença de líquido livre na cavidade abdominal. Para o tratamento domiciliar foi prescrito amoxicilina + ácido clavulânico durante 5 dias, totalizando 10 dias de tratamento até a alta médica. A análise do conteúdo cístico revelou compatível com transudato.

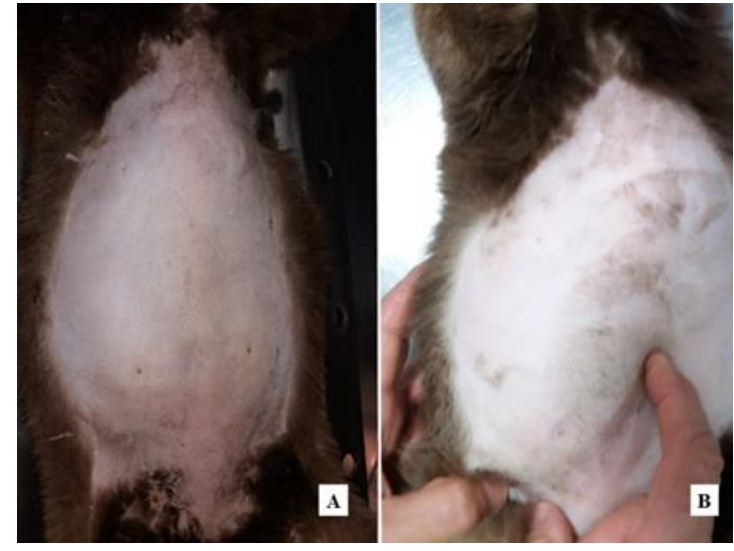

Figura 1. (A) Evidente abdômen distendido; (B) Massa em região cranial do abdômen

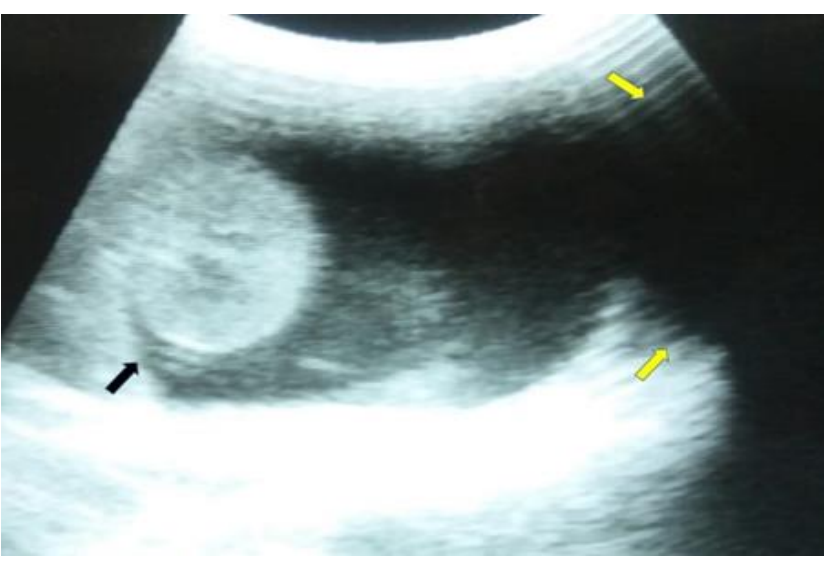

Figura 2. Rim direito (seta preta) envolto por fluido. Pseudocisto perirenal (seta amarela), rim esquerdo.

Consulta de acompanhamento ocorreu 50 dias após o procedimento cirúrgico. Segundo o proprietário, o animal alimentava-se normalmente, fezes e urina sem alterações. Com peso de 4,3 kg e no exame clínico apresentava-se sem alterações. A avaliação do perfil hematológico e bioquímico estavam dentro dos valores de referência para a espécie. Na ecografia abdominal não revelou fluido livre na cavidade abdominal ou recidiva.
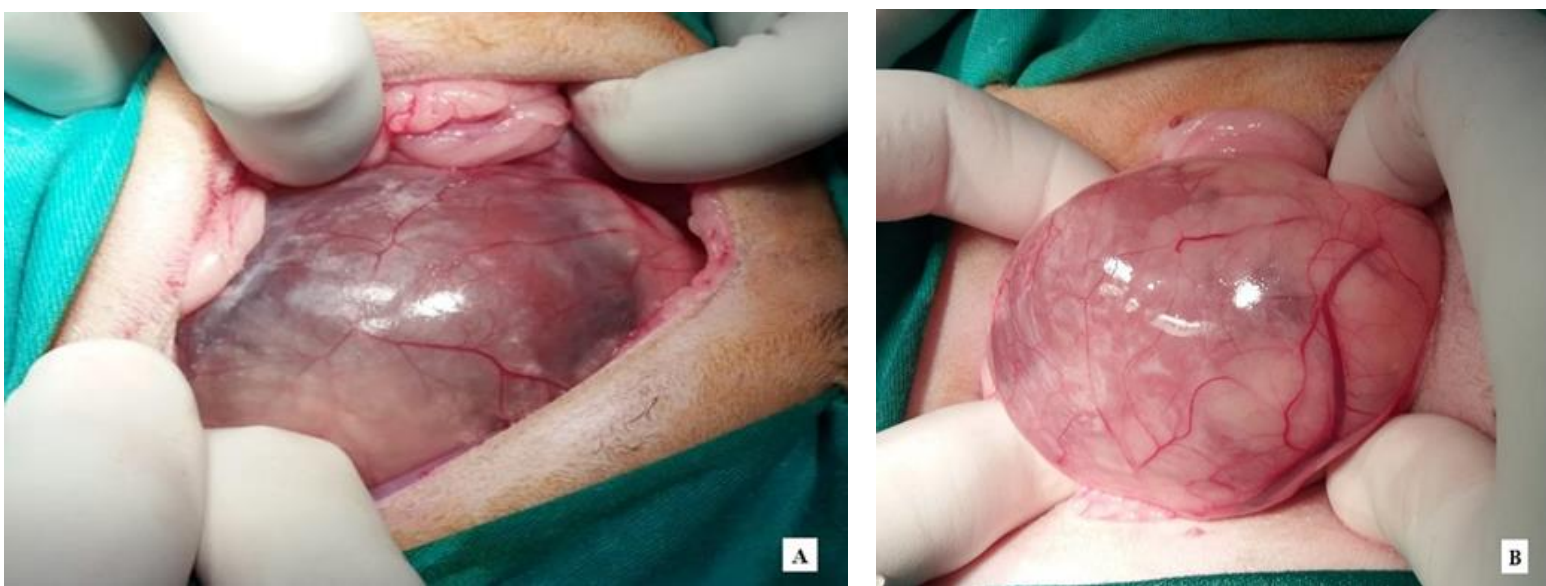

Figura 3. (A) Grande pseudocisto envolvendo o rim direito; (B) Capsula cística com aspecto fino, translucida e vascularizada envolvendo o rim esquerdo.

Após 479 dias o animal retornou para avaliação de rotina. Não apresentava qualquer queixa, pesava $6 \mathrm{~kg}$, na palpação abdominal não havia presença de alterações ou sensibilidade. Exame hematológico e bioquímicos sanguíneo encontravam nos parâmetros normais. No exame ecográfico, não foi observado recidiva, os rins apresentavam-se em topografia habitual e a definição da relação cortico-medular estavam preservadas. No caso apresentado, o procedimento cirúrgico de capsulotomia total bilateral, foi eficaz no tratamento do pseudocisto perirenal. Não observando recidivas e alterações nos perfis bioquímicos e hematológico do animal, durante o período avaliado. 


\section{Discussão}

Pseudocisto perirenal foram relatados no homem (Valli et al., 2011), ovelha (Mutinelli et al., 2005), camundongo (Meyerholz \& Hostetter, 2005), furão (Puerto et al., 1998), cão (Miles \& Jergens, 1992) e gatos (Beck et al., 2000), como apresentado nesse caso.

Os gatos acometidos por pseudocisto perirenal, em sua maioridade são animais idosos. Como apresentado por Beck et al. (2000) em um estudo de 26 casos, onde a média de idade foi de 11 anos. O nosso paciente era um animal adulto com idade de 2 anos no momento do diagnóstico. Contudo há relatos acometendo gatos muito jovens com 2 e 6 meses (Placer \& McManis, 2019; Schaefer et al., 2018). A apresentação clínica dessa desordem podem incluir distensão abdominal, vômito, perda de peso e anorexia (Schaefer et al., 2018). Anorexia e distensão abdominal estavam presentes no gato relatado. Azotenia pode estar presente e ser resultante de uma doença renal primária ou exacerbada pela compressão da pelve renal e dos ureteres proximais (Essman et al., 2000). O aumento da ureia e creatinina estavam presentem somente no momento do diagnóstico do pseudocisto, condizendo com a com compressão das estruturas renais.

O diagnóstico através da ecografia abdominal, revelou imagem compatível com pseudocisto perirenal. O exame de ecografia, permite facilmente visualizar a coleção de fluidos perirenal e exclui outras causas (Debruyn et al., 2012), sendo considerado o método mais útil no diagnóstico dos pseudocistos perirenais (Beck et al., 2000). Além da ecografia outros métodos como radiografia, urografia excretora e cintilografia, podem ser realizadas para estabelecer o diagnóstico de pseudocistos perirrenais (Essman et al., 2000).

A etiologia pode ser multifatorial (Ochoa et al., 1999), como doença renal, neoplasias e congestão venosa (Abdinoor, 1980; Placer \& McManis, 2019). Além de ocorrer sem causa aparente (Castro et al., 2013; Placer \& McManis, 2019), como nesse caso, a causa da formação dos pseudocistos, não foi esclarecida.

O fluido estava acumulado entre a cápsula renal e a parede peritoneal, caracterizando por pseudocisto extracapsular (Beck et al., 2000; Mutinelli et al., 2005). Sendo, o pseudocisto intracapsular é mais frequentemente relatado em gatos, quando comparado com o pseudocisto extracapsular (Beck et al., 2000). A análise da fluido foi compatível com transudato. Sendo o líquido localizado principalmente em gatos com pseudocisto, é o transudato (Debruyn et al., 2012). Mais pode estar presente fluidos com presença de urina, sangue e linfa (Beck et al., 2000).

O tratamento através da drenagem percutânea do fluido do pseudocisto, pode ser realizada, contudo pode ocorrer recidiva, restringindo esse procedimento apenas aos animais que não são candidatos ao procedimento cirúrgico (Beck et al., 2000). O tratamento empregado foi através do procedimento cirúrgico de capsulotomia total. A Ressecção cirúrgica da parede do pseudocisto parece eficaz (Beck et al., 2000), sendo a parede cistica deve ser removida o máximo possível (Ochoa et al., 1999). Pode deixar o pseudoquisto aberto para a cavidade abdominal (Luís et al., 2003). Contudo pode ocorrer o acúmulo de líquido na cavidade abdominal após a capsulotomia, dependendo da origem da fluido (Beck et al., 2000). A omentalização após a capsulotomia pode ser realizada, pois o omento favorece a drenagem fisiológica, eliminar o espaço morto e fornecer uma fonte de neovascularização (Hill \& Odesnik, 2000). No caso relatado, o acúmulo de fluido na cavidade abdominal ou recidiva do pseudocisto, não foi observado em nenhum dos momentos avaliados após capsulotomia.

\section{Conclusão}

As coleções de fluidos ao redor dos rins são pouco relatadas e deve ser considerada um diagnóstico diferencial em animais com distensão abdominal. A técnica cirúrgica de capsulotomia total com omentalização, empregada na resolução de pseudocisto do gato apresentado, demonstrou-se muito eficaz. Não apresentando complicações ou recidivas, proporcionando melhora no quadro clínico e qualidade de vida ao animal no período avaliado. Certamente a não intervenção cirúrgica desse animal, proporcionaria severas alterações fisiológicas e anatômicas, comprometendo a saúde e bem estar do animal.

\section{Agradecimentos}

Os autores agradecem o Programa Equalis de Pós Graduação em Medicina Veterinária, Clínica Veterinária Toca dos Bichos e Fundação para a Ciência e a Tecnologia (FCT), Portugal (SFRH/BD/139319/2018). 


\section{Referências bibliográficas}

Abdinoor, D. J. (1980). Perinephric pseudocysts in a cat. Journal of the American Animal Hospital Association, 16(5), 763-767.

Beck, J. A., Bellenger, C. R., Lamb, W. A., Churcher, R. K., Hunt, G. B., Nicoll, R. G., \& Malik, R. (2000). Perirenal pseudocysts in 26 cats. Australian Veterinary Journal, 78(3), 166-171.

Castro, V. S. P., Castro, J. L. C., Santalucia, S., Raiser, A. G., Caridade, A., Damiani, D., Vaz, C. E. S., Tonin, A. A., Costa, M. M., \& Mazzanti, C. M. (2013). Bilateral Perinephric Pseudocyst in a Cat. Acta Scientiae Veterinariae, 41(1), 1-5.

Debruyn, K., Haers, H., Combes, A., Paepe, D., Peremans, K., Vanderperren, K., \& Saunders, J. H. (2012). Ultrasonography of the feline kidney. Journal of Feline Medicine and Surgery, 14(11), 794 803. https://doi.org/10.1177/1098612X12464461

Essman, S. C., Drost, W. T., Hoover, J. P., Lemire, T. D., \& Chalman, J. A. (2000). Imaging of a cat with perirenal pseudocysts. Veterinary Radiology \& Ultrasound, 41(4), 329-334. https://doi.org/10.1111/j.1740-8261.2000.tb02082.x

Hill, T. P., \& Odesnik, B. J. (2000). Omentalisation of perinephric pseudocysts in a cat. Journal of small animal practice, 41(3), 115-118. DOI:10.1111 / j.1748-5827.2000.tb03177.x

Luís, J. P. S., Vieira, C., Carvalho, A. P., \& Melo, M. (2003). Pseudoquisto renal subcapsular a propósito de dois casos clínicos em gato. Revista Portuguesa de Ciências Veterinárias, 98(548), 211-216.

McCord, K., Steyn, P. F., \& Lunn, K. F. (2008). Unilateral improvement in glomerular filtration rate after permanent drainage of a perinephric pseudocyst in a cat. Journal of Feline Medicine and Surgery, 10(3), 280-283. https://doi.org/10.1016/j.jfms.2007.11.002

Meyerholz, D. K., \& Hostetter, S. J. (2005). Unilateral Perinephric Pseudocyst Secondary to Hydronephrosis in a C57BL/6J Mouse. Veterinary Pathology, 42(4), 496-498. https://doi.org/10.1354/vp.42-4-496

Miles, K. G., \& Jergens, A. E. (1992). Unilateral perinephric pseudocyst of undetermined origin in a dog. Veterinary Radiology \& Ultrasound, 33(5), 277-281. https://doi.org/10.1111/j.17408261.1992.tb00143.x

Mutinelli, F., Vascellari, M., \& Schiavon, E. (2005). Retroperitoneal Perirenal Pseudocyst in a Massese Breed Ram. Journal of Veterinary Diagnostic Investigation, 17(3), 288-290. https://doi.org/10.1177/104063870501700316

Ochoa, V. B., DiBartola, S. P., Chew, D. J., Westropp, J., Carothers, M., \& Biller, D. (1999). Perinephric Pseudocysts in the Cat: A Retrospective Study and Review of the Literature. Journal of Veterinary Internal Medicine, 13(1), 47-55. https://doi.org/10.1111/j.1939-1676.1999.tb02165.x

Placer, M. A., \& McManis, C. (2019). Laparoscopic resection of bilateral perinephric pseudocyst in a pediatric feline patient. Journal of Feline Medicine and Surgery Open Reports, 5(1), 1-4. https://doi.org/10.1177/2055116919850646

Puerto, D. A., Walker, L. M., \& Saunders, H. M. (1998). Bilateral perinephric pseudocysts and polycystic kidneys in a ferret. Veterinary Radiology \& Ultrasound, 39(4), 309-312. https://doi.org/10.1111/j.1740-8261.1998.tb01611.x

Schaefer, G. C., Matesco, V. C., Pereira, P. R., Panziera, W., Driemeier, D., Pavarini, S. P., \& Costa, F. V. A. (2018). Perinephric pseudocyst in a two-month-old female cat. Acta Scientiae Veterinariae, 46, 3.

Valli, R., Piana, S., Capodanno, I., \& Cavazza, A. (2011). Diffuse Large B-Cell Lymphoma Associated With Chronic Inflammation Arising in a Renal Pseudocyst. International Journal of Surgical Pathology, 19(1), 117-119. https://doi.org/10.1177/1066896910391253.

Recebido: 10 de julho, 2020.

Aprovado: 10 de agosto, 2020.

Disponível online: 6 de outubro, 2020.
Licenciamento: Este artigo é publicado na modalidade Acesso Aberto sob a licença Creative Commons Atribuição 4.0 (CC-BY 4.0), a qual permite uso irrestrito, distribuição, reprodução em qualquer meio, desde que o autor e a fonte sejam devidamente creditados. 